\title{
FURTHER GEOMETRY OF THE MEAN CURVATURE ONE-FORM AND THE NORMAL PLANE FIELD ONE-FORM ON A FOLIATED RIEMANNIAN MANIFOLD
}

\author{
GRANT CAIRNS and RICHARD H. ESCOBALES, JR.
}

(Received 24 March 1995)

Communicated by R. Bartnik

\begin{abstract}
For foliations on Riemannian manifolds, we develop elementary geometric and topological properties of the mean curvature one-form $\kappa$ and the normal plane field one-form $\beta$. Through examples, we show that an important result of Kamber-Tondeur on $\kappa$ is in general a best possible result. But we demonstrate that their bundle-like hypothesis can be relaxed somewhat in codimension 2 . We study the structure of umbilic foliations in this more general context and in our final section establish some analogous results for flows.
\end{abstract}

1991 Mathematics subject classification (Amer. Math. Soc.): primary 57R30; secondary 53C25. Keywords and phrases: foliation, mean curvature.

\section{Introduction}

In this paper we study the geometry of the mean curvature one-form $\kappa$ and the normal plane field one-form $\beta$ in the setting of a foliation on a Riemannian manifold. The rich properties of $\kappa$ have been discussed by many authors ([17, 28, 11]), while $\beta$ has been discussed in $([28,16])$. Much of the work on $\kappa$ has been developed in the context of bundle-like foliations, which were an important focus for work on foliations in the 1980's. The bundle-like hypothesis is a severe restriction both on the dynamical behaviour of the foliation, and on the topology of the ambient manifold; for example, their leaf closures are always manifolds [20] and for bundle-like flows, all the Pontryagin numbers are zero [9]. Our purpose has been to further expound on

This paper was completed while the second author was at La Trobe University on sabbatical leave from Canisius College. He wishes to thank La Trobe for its hospitality in making his stay there both pleasant and possible and both institutions for their support.

(C) 1997 Australian Mathematical Society 0263-6115/97 \$A2.00+0.00 
the properties of these forms under weak geometric assumptions. In particular, our main motivation has been to remove the bundle-like hypothesis, where this proves possible.

In Section 1 we recall $\kappa$ and $\beta$ and a number of related concepts. If $X$ and $Y$ are basic vector fields transverse to our foliation and $L$ is a leaf of the foliation, we give a new and simple proof of $d \kappa(X, Y)=-\operatorname{div}_{L} \mathscr{V}[X, Y]$ where $\mathscr{V}[X, Y]$ denotes the leaf component of the Lie bracket (Theorem 1.3).

In Section 2, we adapt the formalism so beautifully expounded in [29] and develop some simple consequences. Now Kamber and Tondeur have shown that for a compact Riemannian manifold with bundle-like foliation, if the associated mean curvature oneform $\kappa$ is a basic, then in fact $\kappa$ is closed [18], [28, p. 150]. This is an important technical point, both in the work of [18] and [13]. Generally speaking, one could say that this step gives real geometric meaning to the hypothesis that the foliation is tense. For example, for a bundle-like flow $\mathscr{F}$, if $\kappa$ is closed, then $\mathscr{F}$ is locally spanned by Killing vectors [13]. In Example 2.4, we show that in general Kamber and Tondeur's compactness hypothesis cannot be dropped, and in Example 2.5, we show that in general their bundle-like hypothesis cannot be dropped. Nevertheless, in Theorem 2.6 we are able to replace the condition on the bundle-like metric with a condition on the transverse volume element provided the codimension of the foliation is 2 .

In Proposition 2.9 we interpret what it means for $d^{2,-1} \beta=0$ while in Proposition 2.10 we show that $d^{2,-1} \beta \neq 0$ represents an obstruction to the integrability of the distribution orthogonal to the leaves, provided $\kappa$ is horizontally closed. Theorem 2.11 is a technical result which tells the story of allowable metric deformations when $\kappa$ and $\beta$ are exact.

In Section 3 we show that if the leaves of a foliation are totally umbilic and $X$ and $Y$ are local basic vector fields transverse to the leaves, then $\mathscr{V}[X, Y]$ is an infinitesimal conformal transformation along the leaves of the foliation (Proposition 3.2). When additionally the foliation codimension is 2 and the manifold admits a basic transverse volume form, we show $\mathscr{V}[X, Y]$ is really globally defined and has rich geometric properties (Theorem 3.3). In the spirit of the earlier part of the paper, we do not require that the metric of the ambient manifold be bundle-like for our results to obtain.

In Section 4 we study flows on a Riemannian manifold in a setting somewhat more general than the bundle-like situation. When $\kappa$ is closed and $X$ and $Y$ are basic $\mathscr{V}[X, Y]$ is always an infinitesimal isometry along the leaves of a flow. When the metric on the ambient manifold is bundle-like we have a generalization of the Gromoll-Grove integrability Lemma for metric flows on the round sphere [13]. This is our Proposition 4.2. Our proof mimics theirs but uses an equation of O'Neill in a different way. 


\section{Section 1}

Throughout this paper all objects and maps are assumed to be of class $C^{\infty}$. We will consider throughout this paper a transversally oriented foliation $\mathscr{F}$ on an oriented, connected Riemannian manifold $(M, g)$ without boundary. As usual, the subbundle $\mathscr{V}$ of the tangent bundle $T M$ is the distribution tangent to $\mathscr{F}$ while $\mathscr{H}$ denotes the orthogonal complement of $\mathscr{V}$ in $T M$ determined by $g$. When there is no danger of confusion, we denote by $\mathscr{V}$ (respectively $\mathscr{H}$ ) the projection $T M \rightarrow \mathscr{V}$ (respectively, the projection $T M \rightarrow \mathscr{H}$ ). A vector field $E$ on $M$ is called vertical if $\mathscr{V} E=E$ and $E$ is horizontal if $\mathscr{H} E=E$. Every vector field $F$ can be written as the direct sum of a vertical and horizontal field: $F=\mathscr{V} F+\mathscr{H} F$.

Let $\left\{V_{1}, V_{2}, \ldots, V_{p}\right\}$ denote a local orthonormal frame for $\mathscr{V}$, where $p=\operatorname{dim} \mathscr{V}$ and let $\left\{X_{1}, X_{2}, \ldots, X_{q}\right\}$ denote a local orthonormal frame of $\mathscr{H}$, where $q=\operatorname{dim} \mathscr{H}$. Then $p+q=n=\operatorname{dim} M$. The tension field $\tau$ of the foliation is defined by $\tau=\sum_{i=1}^{p} \mathscr{H} \nabla_{V_{i}} V_{i}$, where $\nabla$ is the Levi-Civita connection on $M$. We denote by $\omega$ the volume form of the leaves: $\omega\left(E_{1}, E_{2}, \ldots, E_{p}\right)=\operatorname{det}\left(g\left(V_{i}, E_{j}\right)\right)$ and by $\mu$ the transverse volume form $\mu\left(E_{1}, E_{2}, \ldots, E_{q}\right)=\operatorname{det}\left[g\left(X_{i}, E_{j}\right)\right]$.

DEFINITION 1.1. The mean curvature 1 -form $\kappa$ of $\mathscr{F}$ is the 1 -form dual to $\tau$ :

$$
\kappa(E)=g(\tau, E)
$$

for any vector field $E$ on $M$. One has the following equivalent definition of $\kappa$ :

$$
\kappa(E)= \begin{cases}-\left(\mathscr{L}_{E} \omega\right)\left(V_{1}, V_{2}, \ldots, V_{p}\right), & \text { if } E \text { is horizontal } \\ 0, & \text { if } E \text { is vertical, }\end{cases}
$$

where $\mathscr{L}_{E} \omega$ denotes the Lie derivative of $\omega$ with respect to $E$. The equivalence of (i) and (ii) is well known: see for example Tondeur [28, p. 66]. Condition (ii) may be rephrased as [28, page 67]:

$$
\kappa(E)= \begin{cases}\sum_{i=1}^{p} g\left(\left[E, V_{i}\right], V_{i}\right), & \text { if } E \text { is horizontal } \\ 0, & \text { if } E \text { is vertical. }\end{cases}
$$

DEFINITION 1.2. Recall (see [20]) that a vector field $E$ on $M$ is said to be foliate provided for every vertical vector field $W$, one has that $[E, W]$ is vertical. Thus a foliate vector field is an infinitesimal symmetry of $\mathscr{F}$. Let $\left\{U_{a}, f_{\alpha}\right\}_{\alpha \in \Lambda}$ be an atlas on $M$ with submersions $f_{\alpha}: U_{\alpha} \rightarrow f_{\alpha}\left(U_{\alpha}\right) \subseteq \mathbb{R}^{q}$ defining $\mathscr{F}$, with the usual compatibility conditions. Then a vector field $E$ on $M$ is foliate if it projects under each map $f_{\alpha}$ to give a vector field on $f_{\alpha}\left(U_{\alpha}\right)$. We say that $E$ is basic if it is foliate and horizontal. A form $\gamma$ on $M$ is basic provided on each $U_{\alpha}$, one has $\gamma=f_{\alpha}^{*} \gamma_{\alpha}$ where $\gamma_{\alpha}$ is a form 
of the same degree on $f_{\alpha}\left(U_{\alpha}\right)$. Note that $\alpha$ is basic if $\mathscr{L}_{W} \alpha=0$ for all vertical $W$, or equivalently $i_{W} \alpha=0=i_{W} d \alpha$ for all vertical $W$, where $i_{W}$ denotes the interior product. We say the metric $g$ on $M$ is bundle-like with respect to $\mathscr{F}$ provided each of the $f_{\alpha}$ is a Riemannian submersion onto its image. By common abuse of language, $\mathscr{F}$ is said to be bundle-like in this situation.

Notice that $\mathscr{F}$ is bundle-like if its leaves are locally equidistant. Equivalently, this means that the horizontal bundle $\mathscr{H}$ has local orthonormal bases comprised of basic vector fields. If $g$ is bundle-like, then $g$ establishes a duality between basic vector fields and basic forms.

We are now in a position to give a simple proof of the following formula mentioned in the introduction. The original derivation in the appendix to [12] ran 4 pages. The simplicity of the proof below results from the Jacobi identity. Here $\operatorname{div}_{L} \mathscr{V}$ denotes the divergence along the leaf $L$ of $\mathscr{F}$.

THEOREM 1.3. For basic fields $X$ and $Y$, one has $d \kappa(X, Y)=-\operatorname{div}_{L} \mathscr{V}[X, Y]$. In particular, when $\kappa$ is closed, $\mathscr{V}[X, Y]$ leaves invariant the induced volume form of the leaves.

Proof. Since $X$ and $Y$ are basic, $\left[X, V_{i}\right]=\sum A_{i j} V_{j}$ and $\left[Y, V_{i}\right]=\sum B_{i j} V_{j}$. Then $d \kappa(X, Y)=X \kappa(Y)-Y \kappa(X)-\kappa([X, Y])$. So by (iii) of Definition 1.1, this becomes

$$
d \kappa(X, Y)=X \sum B_{i i}-Y \sum A_{i i}-\sum_{i} g\left(\left[\mathscr{H}[X, Y], V_{i}\right], V_{i}\right) .
$$

But

$$
\begin{aligned}
\sum_{i} g([ & \left.\left.\mathscr{H}[X, Y], V_{i}\right], V_{i}\right)=\sum_{i} g\left(\left[[X, Y], V_{i}\right], V_{i}\right)-\sum_{i} g\left(\left[\mathscr{V}[X, Y], V_{i}\right], V_{i}\right) \\
= & \sum_{i} g\left(\left[[X, Y], V_{i}\right], V_{i}\right)+\operatorname{div}_{L} \mathscr{V}[X, Y] \\
= & \sum_{i} g\left(\left[\left[X, V_{i}\right], Y\right], V_{i}\right)+\sum_{i} g\left(\left[X,\left[Y, V_{i}\right]\right], V_{i}\right)+\operatorname{div}_{L} \mathscr{V}[X, Y] \\
= & \sum_{i} g\left(\left[\sum_{j} A_{i j} V_{j}, Y\right], V_{i}\right)+\sum_{i} g\left(\left[X, \sum_{j} B_{i j} V_{j}\right], V_{i}\right)+\operatorname{div}_{L} \mathscr{V}[X, Y] \\
= & \sum_{i} g\left(\sum_{j} A_{i j}\left[V_{j}, Y\right], V_{i}\right)-\sum_{i} g\left(\sum_{j}\left(Y A_{i j}\right) V_{j}, V_{i}\right) \\
& +\sum_{i} g\left(\sum_{j}\left(X B_{i j}\right) V_{j}, V_{i}\right)+\sum_{i} g\left(\sum_{j} B_{i j}\left[X, V_{j}\right], V_{i}\right)+\operatorname{div}_{L} \mathscr{V}[X, Y] \\
= & -\sum_{i} g\left(\sum_{j, k} A_{i j} B_{j k} V_{k}, V_{i}\right)-Y \sum_{i} A_{i i} \\
& +X \sum_{i} B_{i i}+\sum_{i} g\left(\sum_{j, k} B_{i j} A_{j k} V_{k}, V_{i}\right)+\operatorname{div}_{L} \mathscr{V}[X, Y] \\
= & -Y \sum_{i} A_{i i}+X \sum_{i} B_{i i}+\operatorname{div}_{L} \mathscr{V}[X, Y] .
\end{aligned}
$$

Hence $d \kappa(X, Y)=-\operatorname{div}_{L} \mathscr{V}[X, Y]$, as claimed. 
DEFINITION 1.4. Recall that

(a) $(M, g, \mathscr{F})$ is harmonic if $\kappa=0$.

(b) $(M, g, \mathscr{F})$ has constant mean curvature if $\kappa(\tau)$ is constant on the leaves of $\mathscr{F}$.

(c) $(M, g, \mathscr{F})$ is mean curvature invariant provided $\tau$ is basic.

(d) $(M, g, \mathscr{F})$ is tense if $\kappa$ is basic.

REMARK 1.5. Since $\kappa$ annihilates vertical vector fields, $\kappa$ is basic if and only if $d \kappa(X, W)=0$ for all vertical $W$ and horizontal $X$ (see [12, Lemma 1.7]).

Corresponding to $\tau$, there is another vector field $\tau^{\perp}$ defined as follows. For a local orthonormal frame $\left\{X_{1}, X_{2}, \ldots, X_{q}\right\}$ of $\mathscr{H}$, one sets $\tau^{\perp}=\sum_{i=1}^{q} \mathscr{V} \nabla_{X_{i}} X_{i}$. If $\tau^{\perp} \equiv 0$, we say the normal plane field is minimal.

DEFINITION 1.6. The normal plane field one-form $\beta$ is the 1 -form dual to $\tau^{\perp}$ :

$$
\beta(E)=g\left(\tau^{\perp}, E\right)=\sum g\left(\mathscr{V} \nabla_{X_{i}} X_{i}, E\right) .
$$

Hence (see [28, p. 71])

$$
\beta(E)= \begin{cases}-\left(\mathscr{L}_{E} \mu\right)\left(X_{1}, \ldots, X_{q}\right), & \text { for vertical } E \\ 0, & \text { for horizontal } E,\end{cases}
$$

where $\mu$ is the volume element on $\mathscr{H}$, and equivalently

$$
\beta(E)= \begin{cases}\sum_{i=1}^{q} g\left(\left[E, X_{i}\right], X_{i}\right), & \text { for vertical } E \\ 0, & \text { for horizontal } E .\end{cases}
$$

COROLLARY 1.7. If $\beta \equiv 0$, then for basic vectors fields $X$ and $Y$,

$$
d \kappa(X, Y)=-\operatorname{div}_{M} \mathscr{V}[X, Y]
$$

PROOF. Given Theorem 1.3, it suffices to note that

$$
\begin{array}{rl}
\operatorname{div}_{M} & \mathscr{V}[X, Y]=\operatorname{div}_{L} \mathscr{V}[X, Y]+\sum_{j=1}^{q} g\left(\nabla_{X} \mathscr{V}[X, Y], X_{j}\right) \\
& =\operatorname{div}_{L} \mathscr{V}[X, Y]-\sum_{j=1}^{q} g\left(\mathscr{V}[X, Y], \nabla_{X_{j}} X_{j}\right)=\operatorname{div}_{L} \mathscr{V}[X, Y]-g\left(\mathscr{V}[X, Y], \tau^{\perp}\right)
\end{array}
$$

REMARK 1.8. (i) There are various commonly used names for the conditions in the above definitions. In particular, bundle-like foliations are called Riemannian foliations. The terminology 'harmonic foliation' was introduced by Kamber and Tondeur: in codimension 1, a foliation is harmonic if its canonical projection $T M \rightarrow$ $\mathscr{H}$ is a harmonic 1-form. Harmonic foliations are known as minimal foliations [23], 
since the leaves of harmonic foliations are minimal submanifolds. Note that condition $\beta \equiv 0$ just says that $\mu$ is a basic form. Foliations verifying this condition are said to have 'minimal normal plane field' [16] or to have 'a holonomy invariant transverse volume form' [28, p. 71]. Tense foliations [17] are also called 'isoparametric' [13]. Constant mean curvature foliations are also called 'tense' foliations by some authors. Mean curvature invariant foliations were introduced only recently by Walczak in [30]; this notion coincides with that of tense foliations when the metric is bundle-like, but in general it is quite different.

(ii) All of the conditions in Definition 1.4 are genuinely weak geometric conditions. Indeed, one of the fundamental methods of constructing examples of foliations is the method of suspension: here one has a group homomorphism $\phi: \pi_{1}(B) \rightarrow \operatorname{Diff}(F)$, from the fundamental group of one manifold $B$ to the group of diffeomorphisms of another, $F$. Then $\pi_{1}(B)$ acts naturally on $F \times \hat{B}$, where $\hat{B}$ is the universal cover of $B$, and the quotient $F \times{ }_{\phi} B$ is a fibre bundle over $B$ with fibre $F$, called the suspension of $\phi$. Importantly, the second factor in $F \times \hat{B}$ induces a foliation on $F \times_{\phi} B$ whose leaves are covering spaces of $B$. The dynamics of this foliation are precisely the dynamics of the discrete group action of $\pi_{1}(B)$ on $F$, so it can be extremely complicated. Since these foliations are transverse to the fibers of the fibration $F \times_{\phi} B \rightarrow B$, they are all harmonic (for an appropriate choice of metric).

More generally, according to [26], a codimension 1 foliation $\mathscr{F}$ on a compact manifold $M$ is harmonic if and only if every leaf of $\mathscr{F}$ meets a closed transversal. The higher codimensional harmonic foliations have been given homological characterizations by Sullivan [27] and Haefliger [14]. For bundle-like foliations, another cohomological characterization of harmonicity was conjectured by Y. Carrière. This was the subject of many papers (see [25]) and was finally proven in [19]. Recently it was shown in [10] that for bundle-like foliations on compact manifolds, there is always a metric for which the foliation is tense. An example of a foliation which is not tense is given by the Reeb foliation (see the Corollary to the proof of 2.11 below).

\section{Section 2}

We follow Tondeur in $([28,29])$. Let $\Omega^{\mu, v}=\Gamma\left(\wedge^{\mu} \mathscr{H}^{*} \otimes \wedge^{\nu} \mathscr{V}^{*}\right)$. The non-zero range of the groups $\Omega^{\mu, \nu}$ is $0 \leq \mu \leq q$ and $0 \leq \nu \leq p$ where $p=\operatorname{dim} \mathscr{V}$ and $q=\operatorname{dim} \mathscr{H}$. Then $d=d^{0,1}+d^{1,0}+d^{2,-1}$ where $d^{i, j}: \Omega^{\mu, \nu} \rightarrow \Omega^{\mu+i, \nu+j}$. From the identity $d^{2}=0$, one has

$$
\begin{aligned}
& d^{0,1} d^{0,1}=0=d^{2,-1} d^{2,-1} \\
& d^{1,0} d^{0,1}+d^{0,1} d^{1,0}=0 \\
& d^{2,-1} d^{1,0}+d^{1,0} d^{2,-1}=0 \\
& d^{2,-1} d^{0,1}+d^{0,1} d^{2,-1}+d^{1,0} d^{1,0}=0 .
\end{aligned}
$$


Now consider $(M, \mathscr{F}, g)$ with vertical volume element $\omega$ as above.

LEMMA 2.1. $d^{1,0}(-\omega)=\kappa \wedge \omega$.

PROOF. This is immediate from Definition 1.1(ii). See [28, p. 66].

Recall that if $(M, g, \mathscr{F})$ is tense and $\mathscr{F}$ has codimension 1 , then $\kappa$ is closed [12]. Generalizing, one has:

LEMMA 2.2. If $(M, g, \mathscr{F})$ is tense and if $\mathscr{H}$ is integrable, then $\kappa$ is closed.

PROOF. If $\mathscr{H}$ is integrable, then $d^{2,-1} \omega=0$. Now one clearly has $d^{0,1} \omega=0$. So by (D4) one has $d^{1,0} d^{1,0} \omega=0$. But by Lemma 2.1 , we see $d^{1,0}(\kappa \wedge \omega)=0$. Thus

$$
\left(d^{1,0} \kappa\right) \wedge \omega=\kappa \wedge\left(d^{1,0} \omega\right)=-\kappa \wedge \kappa \wedge \omega=0 .
$$

But $d^{1,0} \kappa \wedge \omega=0$ implies $d^{1,0} \kappa=0$. Finally, since $\kappa$ is basic, $d^{0,1} \kappa=0$. Thus $d \kappa=0$ as claimed.

For the next lemma, we suppose that all the leaves of $\mathscr{F}$ are dense. The significance of this hypothesis is that it implies that every basic form on $M$ is either nowhere zero or identically zero.

LEMMA 2.3. If $M$ is compact and $(M, g, \mathscr{F})$ is tense and all the leaves are dense, then

(a) if $\mathscr{F}$ has codimension 1 , then $(M, g, \mathscr{F})$ is harmonic.

(b) if $\mathscr{F}$ has codimension 2, then $\kappa$ is a closed 1-form.

PROOF. (a) Since $\mathscr{F}$ has codimension 1, we have $d^{2,-1} \omega=0$. Since $d^{0,1} \omega=0$, we have $d \omega=d^{1,0} \omega=-\kappa \wedge \omega$, by Lemma 2.1. So $\kappa \wedge \omega$ is exact. Thus, $\int_{M} \kappa \wedge \omega=0$. It follows that $\kappa=0$ for some point $q \in M$, so $\kappa \equiv 0$ on the leaf containing $q$. Consequently, since $\kappa$ is basic and the leaves of $\mathscr{F}$ are dense, $\kappa \equiv 0$.

(b) Using $d^{0,1} \omega=0$ and Lemma 2.1, one has

$$
\begin{aligned}
\kappa \wedge d \omega & =-\kappa \wedge\left(d^{1,0} \omega+d^{0,1} \omega+d^{2,-1} \omega\right) \\
& =-\kappa \wedge\left(d^{1,0} \omega+d^{2,-1} \omega\right)=-\kappa \wedge d^{2,-1} \omega
\end{aligned}
$$

But $\kappa \wedge d^{2,-1} \omega \in \Omega^{3, p-1}$ where $p=\operatorname{dim} \mathscr{F}$. Since $\mathscr{F}$ has codimension $2, \kappa \wedge d \omega=0$. Thus, $d \kappa \wedge \omega=d(\kappa \wedge \omega)$. Since $d(\kappa \wedge \omega)$ is an $n$-form, we have by Stokes' Theorem, $\int_{M} d \kappa \wedge \omega=0$. Since $d \kappa$ is basic, $d \kappa \equiv 0$ as required. 
Now in the case that $(M, g, \mathscr{F})$ is a bundle-like foliation with $M$ compact, Kamber and Tondeur have shown that if $\kappa$ is basic, then $\kappa$ is closed [17], [28, pp. 150-151].

EXAMPLES 2.4. The following example shows if the compactness hypothesis on $M$ is removed $\kappa$ basic does not imply that $\kappa$ is closed. Let $M$ be a Lie group with Lie algebra generators $X, Y, V, W$ subject to the following relations:

$$
[X, Y]=Y+V, \quad[V, W]=W, \quad[Y, W]=-W .
$$

Then the distribution on $M$ spanned by $V$ and $W$ is completely integrable and hence gives rise to a foliation $\mathscr{F}$. Let $g$ be the left invariant metric on $M$ with respect to which $X, Y, V$ and $W$ are orthonormal. Then $\mathscr{F}$ is bundle-like with respect to this metric and $X$ and $Y$ are basic vector fields. The mean curvature one form $\kappa$ of $\mathscr{F}$ is the 1-form dual to $-Y$; it is basic but not closed.

EXAMPLES 2.5. This example shows that Kamber and Tondeur's result doesn't hold without the bundle-like hypothesis. First consider the following basis for $s l(3, \mathbb{R})$ :

$$
\begin{array}{llll}
E_{1}=\delta_{1,2}, & E_{2}=\delta_{2,1}, & E_{3}=\delta_{1,1}-\delta_{2,2} & E_{4}=\delta_{1,3}, \\
E_{5}=\delta_{3,1}, & E_{6}=\delta_{2,3}, & E_{7}=\delta_{3,2}, & E_{8}=\delta_{1,1}-\delta_{3,3},
\end{array}
$$

where $\delta_{i, j}$ is the $3 \times 3$ matrix which has 1 in the $(i, j)$ position and 0 elsewhere. The bracket relations for this algebra are given in Table 1 . The vector fields $\left\{E_{1}, E_{3}\right\}$ span a foliation $\tilde{F}$ on $S L(3, \mathbb{R})$. Now $S L(3, \mathbb{R})$ has a uniform lattice, say $\Gamma[3]$. The left invariant fields $E_{1}, \ldots, E_{8}$ induce vector fields on $M=\Gamma \backslash S L(3, \mathbb{R})$, which we will denote by the same letters. Let $g$ be the Riemannian metric on $M$ for which the fields $E_{1}, \ldots, E_{8}$ are orthonormal and let $F$ be the foliation induced by $\tilde{F}$ on $M$. While $\left\{E_{2}, E_{4}, E_{5}, E_{6}, E_{7}, E_{8}\right\}$ are all horizontal fields of unit length, only $E_{8}$ is basic. If $E_{i}$ is in the above list, $i \neq 8$, then either $\left[E_{i}, E_{1}\right]$ or $\left[E_{i}, E_{3}\right]$ has a non-zero horizontal component and hence $E_{i}$ is not basic. Direct calculation shows that $\kappa$ is the 1-form dual to $E_{8}$. In fact, $\kappa$ is basic but not closed since $d \kappa\left(E_{7}, E_{6}\right)=1$. Now taking into account the Kamber-Tondeur result mentioned in the introduction, we see that the metric cannot be bundle-like for our foliation. We might add that Table 1 together with the techniques of Example 2.5 can be used to find many other foliations with varying properties of $\kappa$ and $\beta$.

Despite the above example, the following generalization of Kamber and Tondeur's result obtains if codimension $\mathscr{F}=2$.

THEOREM 2.6. Suppose that $M$ is compact and that $(M, g, \mathscr{F})$ is a codimension 2 foliation with a basic transverse volume form $\mu$. Then $\kappa$ is closed if $\kappa$ is basic. 


\begin{tabular}{|c||c|c|c|c|c|c|c|c|}
\hline & $E_{1}$ & $E_{2}$ & $E_{3}$ & $E_{4}$ & $E_{5}$ & $E_{6}$ & $E_{7}$ & $E_{8}$ \\
\hline \hline$E_{1}$ & 0 & $E_{3}$ & $-2 E_{1}$ & 0 & $-E_{7}$ & $E_{4}$ & 0 & $-E_{1}$ \\
\hline$E_{2}$ & $-E_{3}$ & 0 & $2 E_{2}$ & $E_{6}$ & 0 & 0 & $-E_{5}$ & $E_{2}$ \\
\hline$E_{3}$ & $2 E_{1}$ & $-2 E_{2}$ & 0 & $E_{4}$ & $-E_{5}$ & $-E_{6}$ & $E_{7}$ & 0 \\
\hline$E_{4}$ & 0 & $-E_{6}$ & $-E_{4}$ & 0 & $E_{8}$ & 0 & $E_{1}$ & $-2 E_{4}$ \\
\hline$E_{5}$ & $E_{7}$ & 0 & $E_{5}$ & $-E_{8}$ & 0 & $-E_{2}$ & 0 & $2 E_{5}$ \\
\hline$E_{6}$ & $-E_{4}$ & 0 & $E_{6}$ & 0 & $E_{2}$ & 0 & $E_{8}-E_{3}$ & $-E_{6}$ \\
\hline$E_{7}$ & 0 & $E_{5}$ & $-E_{7}$ & $-E_{1}$ & 0 & $E_{3}-E_{8}$ & 0 & $E_{7}$ \\
\hline$E_{8}$ & $E_{1}$ & $-E_{2}$ & 0 & $2 E_{4}$ & $-2 E_{5}$ & $E_{6}$ & $-E_{7}$ & 0 \\
\hline
\end{tabular}

TABLE 1. The brackets $\left[E_{i}, E_{j}\right]$

PROOF. First, let us present a technical lemma. This played an important role in [8] and we will use it in the next theorem and again in Section 3 below.

LEMMA 2.7. If $(M, g, \mathscr{F})$ is a codimension 2 foliation with a basic transverse volume form $\mu$, then there is a globally defined vertical vector field $V_{\mu}$ defined locally by setting $V_{\mu}=\mathscr{V}[X, Y]$, where $X, Y$ are local basic vector fields satisfying $\mu(X, Y)=1$.

Proof. It suffices to note that if $X^{\prime}$ and $Y^{\prime}$ are basic with $\mu\left(X^{\prime}, Y^{\prime}\right)=1$ on some open set on which both pairs $\{X, Y\}$ and $\left\{X^{\prime}, Y^{\prime}\right\}$ are defined, then

$$
\left(\begin{array}{l}
X^{\prime} \\
Y^{\prime}
\end{array}\right)=B\left(\begin{array}{l}
X \\
Y
\end{array}\right)
$$

where the $B$ is a matrix whose entries are basic functions and which has determinant 1 . A straightforward calculation shows that $\mathscr{V}\left[X^{\prime}, Y^{\prime}\right]=\operatorname{det}(B) \mathscr{V}[X, Y]=\mathscr{V}[X, Y]$.

Now choose local basic vector fields $X$ and $Y$ such that $\mu(X, Y)=1$. By the above lemma, the vector field $V_{\mu}=\mathscr{V}[X, Y]$ has a global meaning. Similarly, since $\kappa$ is basic, $f=d \kappa(X, Y)$ is a globally defined basic function on $M$. In the same way, $d \kappa(f X, Y)$ and $\mathscr{V}[f X, Y]$ have a global meaning. Now because $\mu$ is basic, $\beta=0$ and hence $\tau^{\perp}=0[28$, p. 71$]$. It follows that

$$
d \kappa(f X, Y)=-\operatorname{div}_{L} \mathscr{V}[f X, Y]=-\operatorname{div}_{M} \mathscr{V}[f X, Y],
$$

by Corollary 1.7. Hence

$$
\int_{M} f^{2}=\int_{M} d \kappa(f X, Y)=\int_{M}-\operatorname{div}_{M} \mathscr{V}[f X, Y]=0
$$


by the divergence theorem. Thus, $f^{2} \equiv 0$ on $M$ and so $f \equiv 0$ on $M$. Hence $d^{1,0} \kappa=0$. But $\kappa \in \Omega^{1,0}$ implies $d^{2,-1} \kappa=0$ and $\kappa$ basic implies $d^{0,1} \kappa=0$. Thus $\kappa$ is closed.

We now turn to $\beta$. We first recall:

LEMMA 2.8. For an arbitrary foliation $(M, g, \mathscr{F})$, the 1 -form $\beta$ is $\mathscr{F}$-closed. That $i s, d^{0,1} \beta=0$.

Proof. From Definition 1.6, $d^{0,1} \mu=-\beta \wedge \mu$. So, as $d^{0,1} \circ d^{0,1}=0$ by (D1), one has

$$
0=d^{0,1} \circ d^{0,1} \mu=-d^{0,1} \beta \wedge \mu+\beta \wedge d^{0,1} \mu=-d^{0,1} \beta \wedge \mu-\beta \wedge \beta \wedge \mu .
$$

Hence, $d^{0,1} \beta \wedge \mu=0$. But $d^{0,1} \beta$ is a form of type $(0,2)$ while $\mu$ is one of type $(q, 0)$. Hence, $d^{0,1} \beta=0$.

Note, one could also show that $\beta$ is $\mathscr{F}$-closed using 1.6 (iii) and imitating the technique of Theorem 1.3.

From Theorem 1.3 we know if $X$ and $Y$ are basic then $d \kappa(X, Y)=-\operatorname{div}_{L} \mathscr{V}[X, Y]$ and so from [11], many nice geometric properties obtain. A natural question to ask, is when does $d \kappa(X, Y)=-\operatorname{div}_{M} \mathscr{V}[X, Y]$ ? As noted in [11, Theorem 1.4], this occurs when $(M, g, \mathscr{F})$ is bundle-like. More generally, by Corollary 1.7 of this paper, if $\beta \equiv 0$, then $d \kappa(X, Y)=-\operatorname{div}_{M} \mathscr{V}[X, Y]$. The following observation along the same lines is elementary.

PROPOSITION 2.9. If $d^{2 .-1} \beta=0$, then $d \kappa(X, Y)=-\operatorname{div}_{M} \mathscr{V}[X, Y]$ for any basic vector fields $X$ and $Y$. In particular, if $\kappa$ is closed (or more generally, $d^{1,0} \kappa=0$ ) and $d^{2,-1} \beta=0$, then $\mathscr{V}[X, Y]$ leaves invariant the volume form of the ambient manifold.

PROOF. It suffices to note that

$$
\begin{aligned}
\operatorname{div}_{M} \mathscr{V}[X, Y] & =\operatorname{div}_{L} \mathscr{V}[X, Y]+\sum_{j=1}^{q} g\left(X_{j}, \nabla_{X_{j}} \mathscr{V}[X, Y]\right) \\
& =-d \kappa(X, Y)-\sum_{j=1}^{q} g\left(\nabla_{X_{j}} X_{j}, \mathscr{V}[X, Y]\right) \\
& =-d \kappa(X, Y)-\beta(\mathscr{V}[X, Y])=-d \kappa(X, Y)+d^{2,-1} \beta(X, Y)
\end{aligned}
$$

Notice that this same calculation gives:

PRoposition 2.10. Suppose $d^{1,0} \kappa=0$ and $d^{2,-1} \beta \neq 0$. Then $\operatorname{div}_{M} \mathscr{V}[X, Y] \neq 0$ for some basic fields $X$ and $Y$. In particular, $\mathscr{H}$ is not integrable. 
Because $d^{0,1} \beta=0$, the 1 -form $\beta$ defines a member of $H^{1}(L)$, for every leaf $L$. Because $\beta$ is globally defined on $M$, it makes sense to consider what happens when $\beta$ is trangressive; that is, when $d \beta$ is basic (see $[4$, p. 247]). Notice that in this case $d \beta$ is a pure form of type $(2,0)$. So, as $d \beta=d^{1,0} \beta+d^{2,-1} \beta$, this gives $d \beta=d^{2,-1} \beta$. Hence we can reformulate Proposition 2.10 as follows.

PROPOSITION 2.10*. Suppose $d^{1,0} \kappa=0$ and $\beta$ is transgressive with $d \beta \neq 0$. Then $\mathscr{V}[X, Y] \neq 0$ for some basic vector fields $X$ and $Y$, and so $\mathscr{H}$ is not integrable.

It is sometimes useful for us to have a metric on $M$ so $(M, g, \mathscr{F})$ has $\beta \equiv 0$. In codimension $1, \beta \equiv 0$ if and only if $\mathscr{F}$ is bundle-like with respect to $g$. In the codimension $q$ case, if $\mathscr{H}$ is integrable the vanishing of $\beta$ is just what is required for the leaves of $\mathscr{H}$ to be minimal submanifolds of $M$. We have:

THEOREM 2.11. Let $\mathscr{F}$ be a transversally oriented foliation on an oriented Riemannian manifold $(M, g)$.

(1) If $\beta$ is exact, there exists a new metric $g^{*}$ on $M$ so that the associated normal plane field one-form $\beta^{*}=0$ and the associated mean curvature one-form $\kappa^{*}=\kappa$ and the associated transverse volume element $\mu^{*}$ is basic. If $X$ and $Y$ are basic vector fields, $d \kappa^{*}(X, Y)=-\operatorname{div}_{M} \mathscr{V}[X, Y]$.

(2) If $\beta$ and $\kappa$ are exact, then one can define a new metric $\tilde{g}$ so the associated mean curvature one form $\tilde{\kappa}=0$, and the associated normal plane field one-form $\tilde{\beta}=0$, and so the transverse volume form $\mu$ is basic. In particular, with respect to $\tilde{g}$, the leaves of $\mathscr{F}$ are minimal submanifolds, and if $\mathscr{H}$ is integrable, the leaves of $\mathscr{H}$ are likewise minimal submanifolds of $M$.

PROOF. (1) Suppose $\beta=d f$. Let $h=f / q$ and consider a new metric $e^{2 h} g=g^{*}$. Now if $E$ is horizontal, then by definition $\beta^{*}(E)=\beta(E)=0$. If $E$ is vertical, then from Definition 1.6(iii),

$$
\begin{aligned}
\beta^{*}(E) & =\sum_{i=1}^{q} e^{2 h} g\left(\left[E, e^{-h} X_{i}\right], e^{-h} X_{i}\right)=\sum_{i=1}^{q} e^{h} g\left(\left[E, e^{-h} X_{i}\right], X_{i}\right) \\
& =\sum_{i=1}^{q} e^{h} g\left(E\left(e^{-h}\right) X_{i}+e^{-h}\left[E, X_{i}\right], X_{i}\right) \\
& =-q \cdot E(h)+\sum_{i=1}^{q} g\left(\left[E, X_{i}\right], X_{i}\right) \\
& =-E(f)+\beta(E)=-d f(E)+\beta(E)=0 .
\end{aligned}
$$

So $\beta^{*}=0$ and by $[28$, p. 71$]$, the transverse volume element $\mu^{*}$ is basic. Similarly, if $E$ is vertical, $\kappa^{*}(E)=\kappa(E)$ and if $E$ is horizontal, then by Definition 1.1(iii),

$$
\kappa^{*}(E)=\sum_{i=1}^{p} e^{2 h} g\left(\left[E, e^{-h} V_{i}\right], e^{-h} V_{i}\right)=\kappa(E)-E(f) .
$$


But by definition of $\beta, 0=\beta(Y)=Y f$, so $f$ is annihilated by horizontal fields. So $E(f)=0$ and hence $\kappa^{*}(E)=\kappa(E)$ and $\kappa$ is unchanged by the metric alteration. It follows from 2.9 that $\mathscr{L}_{\mathscr{Y}[X . Y]} \Omega^{*}=0$ when $\kappa^{*}$ is closed.

(2) Suppose $\beta=d f$ and $\kappa=d \sigma$. As in part (1), replacing $g$ by the new metric $e^{2 f / q} g=g^{*}$, one obtains $\beta^{*}=0$ and $\kappa^{*}(E)=\kappa(E)$. Then, in an entirely analogous manner, by replacing $g^{*}$ by the new metric $e^{2 \sigma / p} g^{*}=\tilde{g}$, one obtains $\tilde{\kappa}=0$ and $\tilde{\beta}=\beta^{*}$. Thus $\tilde{\beta}=\tilde{\kappa}=0$. The last part of the theorem then follows immediately when $\mathscr{H}$ is integrable by the definition of $\beta$.

We now recover the following result (see [28, p. 131]).

COROLlARY TO PROOF OF 2.11 Suppose $(M, g, \mathscr{F})$ is a codimension one foliation on a closed 3-manifold with finite fundamental group. Then $\mathscr{F}$ cannot be tense.

PROOF. If $\mathscr{F}$ is tense, then $\kappa$ is closed. So if $H^{1}(M, \mathbb{R})=0$, then $\kappa$ is exact. Mimicing the proof of (2) of the previous theorem, the metric $g$ can be deformed to a new metric $\tilde{g}$ with corresponding mean curvature one-form $\tilde{\kappa}=0$, contradicting a result of Sullivan $[29$, p. 21].

\section{Section 3}

In this section we will deal with foliations $\mathscr{F}$ having leaves which are totally umbilic. This situation has already been treated in [12] and [7] in the case the metric is bundle-like. On compact 3 -manifolds, a recent classification of totally umbilic foliations was given in [5].

Recall a submanifold $L$ of a manifold $M$ is totally umbilic, provided there is a vector field $N$ perpendicular to $L$ such that for each pair of tangent vectors $U$ and $V$, one has $T_{U} V=g(U, V) N$, where $T$ is the second fundamental form. $N$ is called the normal curvature vector field. In our setting $N$ is globally defined on $M$ because $M$ is foliated by a foliation with totally umbilic leaves. As observed in [12], $\tau=p N$ where $p$ is the leaf dimension. Since we will not be working under the assumption that the metric $g$ on $M$ is bundle-like, we need to make severe alterations on the work of [12], if similar results are to be forthcoming. Structure equations similar to those of O'Neill, see Ranjan [24], will not yield what we need because the identity $A_{X} Y=\mathscr{V}[X, Y] / 2$ for $X, Y$ horizontal obtains only when the metric $g$ is bundle-like for $\mathscr{F}$. (Recall that $A$ is the integrability tensor of [22] or [21]: $A_{E} F=\mathscr{V} \nabla_{\mathscr{H} E} \mathscr{H} F+\mathscr{H} \nabla_{\mathscr{H} E} \mathscr{V} F$.) We will use the following well-known identity, which will be applied with $X$ and $Y$ basic and $V$ and $W$ vertical. 
PROPOSITION 3.1. $\left(\mathscr{L}_{[X, Y]} g\right)(V, W)=\mathscr{L}_{X}\left(\mathscr{L}_{Y} g\right)(V, W)-\mathscr{L}_{Y}\left(\mathscr{L}_{X} g\right)(V, W)$, or equivalently,

$$
\begin{aligned}
{[X, Y] g } & (V, W)-g([[X, Y], V], W)-g(V,[[X, Y], W]) \\
= & X\left(\mathscr{L}_{Y} g\right)(V, W)-\left(\mathscr{L}_{Y} g\right)([X, V], W)-\left(\mathscr{L}_{Y} g\right)(V,[X, W]) \\
& -Y\left(\mathscr{L}_{X} g\right)(V, W)+\left(\mathscr{L}_{X} g\right)([Y, V], W)+\left(\mathscr{L}_{X} g\right)(V,[Y, W]) .
\end{aligned}
$$

PROOF. Simply expand both sides. The result follows from the Jacobi identity.

We have the following result.

PROPOSITION 3.2. Let $M$ be a Riemannian manifold with totally umbilic foliation $\mathscr{F}$. Then if $X$ and $Y$ are basic vector fields, $\mathscr{V}[X, Y]$ is an infinitesimal conformal transformation along the leaves of $\mathscr{F}$. If $d \kappa(X, Y)$ is basic, then $\mathscr{V}[X, Y]$ is an infinitesimal homothetic transformation on these leaves. Finally, if $\kappa$ is closed, then $\mathscr{V}[X, Y]$ is an infinitesimal isometry on these leaves.

PROOF. We work locally. Let $V$ and $W$ be vertical vector fields. We will show $\left(\mathscr{L}_{\mathcal{V}[X, Y]} g\right)(V, W)=f g(V, W)$. First note that for every horizontal vector field $Z$ one has

$$
\begin{aligned}
\left(\mathscr{L}_{Z} g\right)(V, W) & =Z g(V, W)-g([Z, V], W)-g(V,[Z, W]) \\
& =g\left(\nabla_{V} Z, W\right)+g\left(V, \nabla_{W} Z\right)=-g\left(Z, T_{V} W\right)-g\left(Z, T_{W} V\right) \\
& =-2 g\left(Z, T_{V} W\right)=-2 g(Z, N) g(V, W) .
\end{aligned}
$$

Then, rewriting Proposition 3.1,

$$
\begin{aligned}
&\left(\mathscr{L}_{\mathscr{H}[X, Y]} g\right)(V, W)+\left(\mathscr{L}_{\mathcal{V}[X, Y]} g\right)(V, W) \\
&= X\left(\mathscr{L}_{Y} g\right)(V, W)-\left(\mathscr{L}_{Y} g\right)([X, V], W)-\left(\mathscr{L}_{Y} g\right)(V,[X, W]) \\
&-Y\left(\mathscr{L}_{X} g\right)(V, W)+\left(\mathscr{L}_{X} g\right)([Y, V], W)+\left(\mathscr{L}_{X} g\right)(V,[Y, W]),
\end{aligned}
$$

gives

$$
\begin{aligned}
-2 g( & \mathscr{H}[X, Y], N) g(V, W)+\left(\mathscr{L}_{\left.\mathcal{Y}^{X}, Y\right]} g\right)(V, W) \\
= & -X\{2 g(Y, N) g(V, W)\}+2 g(Y, N) g([X, V], W)+2 g(Y, N) g(V,[X, W]) \\
& +Y\{2 g(X, N) g(V, W)\}-2 g(X, N) g([Y, V], W)-2 g(X, N) g(V,[Y, W]) \\
= & 2\left[-g(Y, N)\left(L_{X} g\right)(V, W)-g(V, W) X g(Y, N)\right. \\
& \left.+g(X, N)\left(\mathscr{L}_{Y} g\right)(V, W)+g(V, W) Y g(X, N)\right] \\
= & 2[g(Y, N) 2 g(X, N) g(V, W)-g(V, W) X g(Y, N) \\
& -g(X, N) 2 g(Y, N) g(V, W)+g(V, W) Y g(X, N)] \\
= & 2[g(V, W) Y g(X, N)-g(V, W) X g(Y, N)]
\end{aligned}
$$


and so

$$
\left(\mathscr{L}_{\mathcal{V}[X, Y]} g\right)(V, W)=2 g(V, W)[g(\mathscr{H}[X, Y], N)+Y g(X, N)-X g(Y, N)] .
$$

This means that $\mathscr{L}_{\mathscr{Y}_{[X, Y]}} g(V, W)=f g(V, W)$ and so $\mathscr{V}[X, Y]$ is a local infinitesimal conformal transformation on the leaves of $\mathscr{F}$. Finally, note that

$$
\begin{aligned}
\left(\mathscr{L}_{\mathscr{V}[X, Y]} g\right)(V, W) & =g\left(\nabla_{V} \mathscr{V}[X, Y], W\right)+g\left(V, \nabla_{W} \mathscr{V}[X, Y]\right) \\
& =\frac{2}{p} \operatorname{div}_{L} \mathscr{V}[X, Y] g(V, W)=-\frac{2}{p} d \kappa(X, Y) g(V, W)
\end{aligned}
$$

by Theorem 1.3 and the fact $\tau=p N$. The last part of 3.2 follows directly from [11, Theorem 3.1].

We are now in a position to formulate one of the main results of this paper. Note, while the ambient manifold has a Riemannian metric we do not require that the metric be bundle-like for the foliation.

THEOREM 3.3. Let $M$ be an oriented Riemannian manifold with transversally oriented foliation $\mathscr{F}$ of codimension 2 having totally umbilic leaves. Assume $(M, g, \mathscr{F})$ admits a basic transverse volume form $\mu$.

(1) When restricted to a leaf of $\mathscr{F}$, the globally defined vector field $V_{\mu}=\mathscr{V}[X, Y]$, given by Lemma 2.7 , is an infinitesimal conformal transformation along the leaf.

(2) If $\kappa$ is basic, then $\mathscr{V}[X, Y]$ is an infinitesimal homothetic transformation when restricted to a leaf of $\mathscr{F}$.

(3) If $\kappa$ is closed, then $\mathscr{V}[X, Y]$ is in fact an infinitesimal isometry on the leaves of $\mathscr{F}$ and an infinitesimal volume preserving diffeomorphism of $M$. These properties are independent of the choice of representative of the basic cohomology class represented by $\kappa$. In fact, if $\kappa^{*}=\kappa+d \sigma$ where $\sigma$ is a basic function on $M$, then $\left(M, g^{*}, \mathscr{F}\right)$, where $g^{*}$ is the metric associated with the mean curvature one-form $\kappa^{*}$, is totally umbilic and admits a basic transverse volume form.

(4) Suppose each leaf of $\mathscr{F}$ has quasi-negative Ricci curvature and additionally each of the following added conditions obtains:

(i) $M$ is compact.

(ii) $\kappa$ is basic or the leaf dimension of $\mathscr{F}$ is 2 .

Then $\mathscr{H}$ is integrable.

PROOF. (1) follows immediately from Proposition 3.2.

(2) If $\kappa$ is basic, then $d \kappa(X, Y)$ is constant on the leaves of $\mathscr{F}$ and so, by (1), $\mathscr{V}[X, Y]$ is an infinitesimal homothetic transformation when restricted to the leaves of $\mathscr{F}$. 
(3) Because $\mu$ is basic, $\beta=0$ by [28, p. 71]. By Theorem 1.3 and Corollary 1.7 , it follows $\mathscr{V}[X, Y]$ is an infinitesimal isometry along the leaves of $L$ and its diffeomorphisms preserve the volume of $M$. By [11] all of the geometric properties of the leaves persist under the conformally altered metric $g^{*}=e^{-2 \sigma / p} g$, when $\sigma$ is basic.

(4) If $\kappa$ is basic and $M$ is compact, then $\kappa$ is closed by Theorem 2.6 above. Let $\tilde{\nabla}$ be the induced connection on the leaves. Then since $\mathscr{V}[X, Y]$ is conformal on the leaves, one has when $\kappa$ is closed that $\mathscr{V}[X, Y]$ is Killing on each leaf so

$$
\begin{aligned}
\Delta_{L} g(\mathscr{V}[X, Y], \mathscr{V}[X, Y] \\
\quad=\sum_{i=1}^{p} g\left(\tilde{\nabla}_{V_{i}} \mathscr{V}[X, Y], \tilde{\nabla}_{V_{i}} \mathscr{V}[X, Y]\right)-S_{L}(\mathscr{V}[X, Y], \mathscr{V}[X, Y]) \geq 0
\end{aligned}
$$

where $\left\{V_{i}\right\}$ is a local orthonormal frame of $V$ and $S_{L}$ is the Ricci tensor of $L$. Thus, $g(\mathscr{V}[X, Y], \mathscr{V}[X, Y])$ is subharmonic on each leaf. If $M$ is compact, let $p$ be the point where $g(\mathscr{V}[X, Y], \mathscr{V}[X, Y])$ attains its maximum. Then by the maximum principle for subharmonic functions, $\Delta_{L} g(\mathscr{V}[X, Y], \mathscr{V}[X, Y]) \equiv 0$. But the right hand side of $\left.{ }^{* *}\right)$ is positive somewhere on $L$ unless $\mathscr{V}[X, Y] \equiv 0$ on $L$. This means $\mathscr{V}[X, Y] \equiv 0$ on $L$ and hence on $M$ since $p \in L$. Thus, $\mathscr{H}$ is integrable.

In the special case when the leaf dimension is 2, the extra term that arises in the computation of $\Delta_{L} g(\mathscr{V}[X, Y], \mathscr{V}[X, Y])$ on the leaf when $\mathscr{V}[X, Y]$ is just an infinitesimal conformal transformation, disappears by the computation of Bochner himself $[2$, Lemma 2]. The argument above now leads to the conclusion $\mathscr{V}[X, Y] \equiv 0$ without the added requirement that $\kappa$ be closed.

\section{Section 4}

We now consider $(M, g, \mathscr{F})$ when $\mathscr{F}$ is a foliation of leaf dimension 1 (that is, a flow). We make no assumption at first that the metric $g$ is bundle-like. Analogous to Theorem 3.3, we have the following result.

THEOREM 4.1. Let $(M, g)$ be an oriented Riemannian manifold with transversally oriented flow $\mathscr{F}$. Let $X$ and $Y$ be basic vector fields.

(1) If $d \kappa$ is basic, then when restricted to a leaf of $\mathscr{F}, \mathscr{V}[X, Y]$ is a local infinitesimal homothetic transformation.

(2) If $\kappa$ is closed, then when restricted to a leaf of $\mathscr{F}, \mathscr{V}[X, Y]$ is a local infinitesimal isometry. If $\kappa^{*}=\kappa-d \sigma$, where $\sigma$ is basic, then $\mathscr{V}[X, Y]$ remains an infinitesimal isometry along the leaves of $\mathscr{F}$ with respect to the conformally altered metric $g^{*}=e^{2 \sigma} g$ whose associated mean curvature one-form is $\kappa^{*}$. 
(3) If $\kappa$ is exact, $\kappa=d \sigma$, then $\mathscr{F}$ is geodesible. In particular, the leaves of $\mathscr{F}$ are totally geodesic with respect to the metric $g^{*}=e^{2 \sigma} g$.

(4) If $\kappa$ and $\beta$ are both exact, $\kappa=d \sigma$ and $\beta=d f$, then there exists a metric for which $\mathscr{F}$ is geodesic and has minimal normal plane field.

PROOF. (1) If $\kappa$ is basic, then $d \kappa(X, Y)$ is basic and by Theorem $1.3,-d \kappa(X, Y)=$ $\operatorname{div}_{L} \mathscr{V}[X, Y]$ is constant on the leaves of $\mathscr{F}$. So $\mathscr{V}[X, Y]$ is homothetic.

(2) If $d \kappa(X, Y)=0$, the above means $\mathscr{N}[X, Y]$ is a local infinitesimal isometry along each leaf of $\mathscr{F}$. The rest of (2) follows from Theorem 3.1 of [11].

(3) If $\kappa$ is exact, $\kappa=d \sigma$ then $0=\kappa^{*}=\kappa-d \sigma$ is the associated mean curvature one-form for the metric $e^{2 \sigma} g=g^{*}$ by the argument of Theorem 2.11. Now if $\kappa^{*}=0$, this means that the leaves are totally geodesic because they have dimension 1 .

(4) follows from 2.11.

In the special case that $g$ is bundle-like for the flow $\mathscr{F}$ we have the following generalization of a result of [13] for spheres $S^{n}$.

PROPOSITION 4.2. Let $M$ be a complete connected Riemannian manifold with bundle-like flow $\mathscr{F}$. Suppose $\kappa$ is closed and assume that

(i) there is a point $q \in M$ so for all basic $X, Y$ one has $\mathscr{V}[X, Y]=0$ at $q$.

(ii) for all horizontal $X, Y, Z$ and vertical $W, g\left(R_{X Y} Z, W\right)=0$, where $R$ is the curvature tensor on $M$.

Then the orthogonal distribution $\mathscr{H}$ is integrable.

PROOF. The argument mimics that of [13] (the same sort of argument is employed in [1], for example). Clearly the subset $S$ where $\mathscr{H}$ is integrable is closed. Also $q \in S$ so $S$ is non-empty. By 4.1, if $X$ and $Y$ are basic and $\kappa$ is closed, then $\mathscr{V}[X, Y]=a V$ where $a$ is constant on the leaf. Hence $S$ is a union of leaves of $F$. We will show $S$ is open. Let $p \in L$ be any point of $M$. We will show $A_{X} Y$ vanishes along any horizontal geodesic $\gamma$ emanating from $p$. We use \{3\} of O'Neill [22] which says for $X, Y, Z$ horizontal and $V$ vertical,

$$
\begin{aligned}
g\left(R_{X Y} Z, V\right)= & g\left(\nabla_{Z}\left(A_{X} Y\right), V\right)-g\left(A_{\mathscr{H} \nabla_{Z} X} Y, V\right)-g\left(A_{X} \mathscr{H} \nabla_{Z} Y, V\right) \\
& +g\left(A_{X} Y, T_{V} Z\right)-g\left(A_{Y} Z, T_{V} X\right)-g\left(A_{Z} X, T_{V} Y\right) .
\end{aligned}
$$

Here $A$ is the integrability tensor and $T$ is the second fundamental form of the leaves. Suppose $\left\{X_{1}, X_{2}, \ldots, X_{n-1}\right\}$ is an orthonormal family of basic vector fields in a neighbourhood $N$ of $p$. Setting $X=X_{i}, Y=X_{j}, Z=X_{k}, \mathscr{H} \nabla_{X_{i}} X_{j}=\Gamma_{i j}^{k} X_{k}$ and $A_{X_{i}} X_{j}=f_{i j} V$ the above curvature equation and assumption (ii) means that along $\gamma$ we have a system of homogeneous differential equations satisfying the usual 
initial conditions. Since $f_{i j}(p)=0$, the uniqueness theorem for differential equations guarantees $f_{i j} \equiv 0$ along an open interval on $\gamma$ around $p$. This means $S$ is open.

It would be wonderful to have a result like 4.2 in a more general setting for flows which are not bundle-like. While there are structure equations like the equations in [22] (see the paper of Ranjan [24] for these equations), the crucial property $2 A_{X} Y=$ $\mathscr{V}[X, Y]$ fails when the metric is not bundle-like.

\section{References}

[1] K. Abe, 'Applications of a Riccati type differential equation to Riemannian manifolds with totally geodesic distributions', Tôhoku Math.J. 25 (1973), 425-444.

[2] S. Bochner, 'Vector fields and Ricci curvature', Bull. Amer. Math. Soc. 52 (1946), 776-797.

[3] A. Borel, 'Compact Clifford-Klein forms of symmetric spaces', Topology 2 (1963), 111-122.

[4] R. Bott and L. Tu, Differential forms in algebraic topology (Springer, New York, 1982).

[5] M. Brunella and E. Ghys, 'Umbilic foliations and transversely holomorphic flows', J. Differential Geom. 41 (1995), 1-19.

[6] G. Cairns, 'A general description of totally geodesic foliations', Tôhoku Math. J. 381 (1986), $37-55$.

[7] _ ' 'Totally umbilic Riemannian foliations', Michigan Math. J. 37 (1990), 145-159.

[8] G. Caims and E. Ghys, 'Totally geodesic foliations on 4-manifolds', J. Differential Geom. 23 (1986), 241-254.

[9] Y. Carrière, 'Appendix A - Variations on Riemannian flows', in: Riemannian foliations (P. Molino) (Birkhauser, Boston, 1988) pp. 217-234.

[10] D. Domínguez, 'Finiteness and tenseness theorems for Riemannian foliations', preprint.

[11] R. Escobales, 'The mean curvature cohomology class for foliations and the infinitesimal geometry of the leaves', Differential Geom. Appl. 2 (1992), 167-178.

[12] R. Escobales and P. Parker, 'Geometric consequences of the normal curvature cohomology class in umbilic foliations', Indiana Univ. Math. J. 37 (1988), 389-408.

[13] D. Gromoll and K. Grove, 'One-dimensional metric foliations in constant curvature spaces', in: Differential geometry and complex analysis H. E. Rauch memorial volume (eds. I. Chavel and H. M. Farkas) (1985) pp. 165-168.

[14] A. Haefliger, 'Some remarks on foliations with minimal leaves', J. Differential Geom. 15 (1980), 269-284.

[15] — 'Groupoides d'holonomie et classifiants', Astérisque 116 (1984), 98-107.

[16] H. Kamada, 'Foliations on manifolds with positive constant curvature', Tokyo J. Math. 16 (1993), 49-60.

[17] F. Kamber and Ph. Tondeur, 'Foliations and metric', in: Differential geometry (eds. R. Brooks, A. Gray, B. Reinhart) (Birkhauser, Boston, 1983) pp. 103-152.

[18] _ _ 'De Rham-Hodge theory for Riemannian foliations', Math. Ann. 277 (1987), 415-431.

[19] X. Masa, 'Duality and minimality in Riemannian foliations', Comment. Math. Helv. 67 (1992), $17-27$.

[20] P. Molino, Riemannian foliations (Birkhauser, Boston, 1988).

[21] T. Nagano, 'On fibred Riemann manifolds', Sci. Papers College Gen. Ed. Univ. Tokyo 10 (1960), $17-27$. 
[22] B. O'Neill, 'The fundamental equations of a submersion', Michigan Math. J. 13 (1966), 459-469.

[23] G. Oshikiri, 'Mean curvature function of codimension-one foliations', Comment. Math. Helv. 65 (1990), 79-84, II 66 (1991), 512-520.

[24] A. Ranjan, 'Structural equations and an integral formula for foliated manifolds', Geom. Dedicata 20 (1986), 85-91.

[25] V. Sergiescu, 'Appendix B - Basic cohomology and tautness of Riemannian foliations', in: Riemannian foliations (by P. Molino) (Birkhauser, Boston, 1988) pp. 235-248.

[26] D. Sullivan, 'Cycles for the dynamical study of foliated manifolds and complex manifolds', Inventiones Math. 36 (1976), 225-255.

[27] _ 'A homological characterization of foliations consisting of minimal surfaces', Comment. Math. Helv. 54 (1979), 218-223.

[28] P. Tondeur, Foliations on Riemannian manifolds (Springer, New York, 1988).

[29] _ _ ' 'Geometry of Riemannian foliations', Seminar of mathematical sciences, 20 (Keio University, Yokohama, Japan, 1994).

[30] P. Walczak, 'Foliations invariant under the mean curvature flow', Illinois J. Math. 37 (1993), $609-623$.

School of Mathematics

La Trobe University

Melbourne 3083

Australia

e-mail: g.cairns@latrobe.edu.au
Department of Mathematics

Canisius College

Buffalo NY 14208

USA

e-mail: escobalr@gort.canisius.edu 